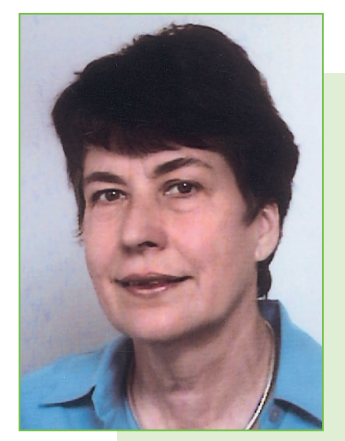

\title{
Besonderheiten der pharmakologischen Prüfung von Phytopharmaka
}

1970 kam ich im Institut für Pharmakologie und Toxikologie in Münster bei Herrn Prof. Dr. F.H. Kemper zum ersten Mal mit pharmakologischen Untersuchungen von Phytopharmaka in Kontakt. Die Untersuchung solcher Zubereitungen wurde in seinem Institut ganz selbstverständlich nach denselben Kriterien vorgenommen, wie sie für Synthetika üblich waren. Anfangs war mir nicht klar, wie sehr diese Einstellung gegenüber pflanzlichen Arzneimitteln unüblich war, schon gar zu der damaligen Zeit. Fragestellungen auf diesem Gebiet haben in den folgenden Jahren bei meiner Arbeit immer mehr Raum eingenommen.

Was ist aber nun so faszinierend an der wissenschaftlichen Arbeit mit Arzneipflanzen? Warum ist die Beschäftigung mit dem Wirknachweis daraus hergestellter Zubereitungen immer wichtiger geworden? Durch die komplexe Zusammensetzung dieser Arzneizubereitungen, bei denen in der Regel der/die Wirkstoffe nicht bekannt sind, ist die pharmakologische Untersuchung ungleich schwieriger als bei Synthetika, sie stellt also eine Herausforderung dar. So sind In-vitro-Untersuchungen als erster Schritt eines Wirknachweises wenig relevant - auch das im Gegensatz zu Synthetika. Bei Synthetika wird nach pharmakokinetischen Untersuchungen in vivo festgestellt, ob die Substanz resorbiert wird, ob ein ausgeprägter First-Pass-Metabolismus vorliegt und welche Plasmaspiegel erreicht werden. So sinnvoll solche Studien mit Einzelstoffen sind - u.a. zum Festlegen eines relevanten Bereiches für die In-vitro-Testung - so wenig Sinn machen sie bei Vielstoffgemischen, deren wirksamer Inhaltsstoff bzw. Inhaltsstoffe unbekannt sind. Bei solchen Vielstoffgemischen ist darüber hinaus unklar, ob der Wirkstoff bereits im Ausgangsmaterial vorliegt oder erst nach metabolischem Umbau in der Leber entsteht, ob es sich also um ein »Prodrug» handelt.

Wie aber soll man bei der pharmakologischen Testung von Vielstoffgemischen vorgehen? In meiner Arbeitsgruppe wird grundsätzlich versucht, die klinische Wirkung in ein Tiermodell zu »übersetzen». Ein Beispiel dafür, wie eine solche Modellsuche durchgeführt werden kann, aber auch welche Irrtümer dabei möglich sind, finden Sie in Heft 3/06. In dem Artikel ging es um einen Wirknachweis für Zubereitungen aus Lycopus europaeus. Solche Zubereitungen wurden und werden traditionell u.a. bei milden Formen der
Schilddrüsenüberfunktion eingesetzt und sollen vor allem die kardialen Wirkungen erhöhter Schilddrüsenhormonspiegel wie Tachykardien bzw. Arrhythmien bessern. Der vermutete Wirkmechanismus eine Senkung der Schilddrüsenhormonspiegel - ließ sich erst bei irrelevant hohen Extraktdosierungen erzielen, die vermutlich weit über dem therapeutischen Bereich lagen.

Was war die Ursache für diese Diskrepanz? War unsere Annahme der klinischen Wirksamkeit von Lycopus-Zubereitungen also falsch oder war das eingesetzte Modell nicht adäquat? Wir haben die Untersuchungen zur Wirkung von Lycopus-Zubereitungen Jahre später wieder aufgenommen und diesmal als primäre Zielparameter Blutdruck, Herzfrequenz und Betarezeptordichte am Herzen der hyperthyreoten Ratte gewählt. Dabei zeigte sich eine ausgeprägte Wirkung auf die Zielparameter selbst in der niedrigsten Dosierung, die wir eigentlich als No-Effect-Level gewählt hatten. Bei Wahl des geeigneten Testmodells ergab sich also eine ganz ausgeprägte Wirkung des untersuchten Phytopharmakons, der klinische Hinweis auf eine besonders ausgeprägte kardiale Wirksamkeit ließ sich im Experiment bestätigen.

Damit möchte ich klarmachen, welche Chancen wir bei der pharmakologischen Untersuchung von Phytopharmaka haben, die lange am Menschen angewendet wurden. Wir können von einer Wirksamkeit am Menschen ausgehen und das ist sehr viel mehr, als wir von neuen Synthetika wissen. Die Berichte über klinische Wirkungen von Phytopharmaka müssen wir unbedingt ernst nehmen und die Testmodelle sehr genau durchdenken, um nicht falsch negative Resultate zu erhalten - schließlich kann ein klinischer Effekt auf unterschiedlichem Wege zustande kommen. Ich wünsche mir eine intensive $\mathrm{Zu}$ sammenarbeit mit Ärzten, die Phytopharmaka anwenden, um deren Erfahrungen in experimentelle Modelle umsetzen zu können. Eine Vielzahl negativer Ergebnisse in experimentellen Modellen dürfte auf die Wahl eines nicht adäquaten Testmodells zurückzuführen sein, nicht auf die Unwirksamkeit des Phytopharmakons. Wie bereits gesagt, die pharmakologische Untersuchung von Phytopharmaka stellt eine faszinierende und vielversprechende Herausforderung dar.

Hilke Winterhoff, Münster 